\title{
Article \\ Searching for Small Molecules as Antibacterials: Non-Cytotoxic Diarylureas Analogues of Triclocarban
}

\author{
Alessia Catalano ${ }^{1, *(\mathbb{D}}$, Domenico Iacopetta $\left.{ }^{2} \mathbb{(}\right)$, Antonio Rosato ${ }^{1}{ }^{\mathbb{D}}$, Lara Salvagno ${ }^{1} \mathbb{D}$, Jessica Ceramella ${ }^{2}$, \\ Francesca Longo ${ }^{1}$, Maria Stefania Sinicropi ${ }^{2,+}+$ ib and Carlo Franchini ${ }^{1, \dagger}$ \\ 1 Department of Pharmacy-Drug Sciences, University of Bari “Aldo Moro”, 70126 Bari, Italy; \\ antonio.rosato@uniba.it (A.R.); lara.salvagno89@gmail.com (L.S.); f.longo21@studenti.uniba.it (F.L.); \\ carlo.franchini@uniba.it (C.F.) \\ 2 Department of Pharmacy, Health and Nutritional Sciences, University of Calabria, 87036 Arcavacata, Italy; \\ domenico.iacopetta@unical.it (D.I.); jessicaceramella@gmail.com (J.C.); s.sinicropi@unical.it (M.S.S.) \\ * Correspondence: alessia.catalano@uniba.it; Tel.: +39-0805442731 \\ + These Authors equally contributed to this work.
}

Citation: Catalano, A.; Iacopetta, D.; Rosato, A.; Salvagno, L.; Ceramella, J.; Longo, F.; Sinicropi, M.S.; Franchini, C. Searching for Small Molecules as Antibacterials: Non-Cytotoxic Diarylureas Analogues of Triclocarban. Antibiotics 2021, 10, 204 https://doi.org/10.3390/ antibiotics 10020204

Academic Editor: Anthony Clarke

Received: 3 February 2021

Accepted: 18 February 2021

Published: 19 February 2021

Publisher's Note: MDPI stays neutral with regard to jurisdictional claims in published maps and institutional affiliations.

Copyright: (c) 2021 by the authors. Licensee MDPI, Basel, Switzerland. This article is an open access article distributed under the terms and conditions of the Creative Commons Attribution (CC BY) license (https:// creativecommons.org/licenses/by/ $4.0 /)$.

\begin{abstract}
Triclocarban (TCC), a broad-spectrum lipophilic antimicrobial agent, is a diarylurea derivative that has been used for more than 60 years as a major ingredient of toys, clothing, food packaging materials, food industry floors, medical supplies and especially of personal care products, such as soaps, toothpaste and shampoo. In September 2016, the U.S. FDA banned nineteen antimicrobial ingredients, including TCC, in over-the-counter consumer antiseptic wash products, due to their toxicity. Withdrawal of TCC has prompted efforts to search for new antimicrobial compounds. In this paper, we present the synthesis and biological evaluation, as antibiotic and non-cytotoxic agents, of a series of diarylureas, analogues of TCC. These compounds are characterized by an intriguingly simple chemistry and can be easily synthesized. Among the synthesized compounds, $\mathbf{1 a b}$ and $\mathbf{1 b c}$ emerge as the most interesting compounds as they show the same activity of TCC (MIC $=16 \mu \mathrm{g} / \mathrm{mL}$ ) against Staphylococcus aureus, and a higher activity than TCC against Enterococcus faecalis (MIC $=32 \mu \mathrm{g} / \mathrm{mL}$ versus MIC $=64 \mu \mathrm{g} / \mathrm{mL}$ ). Moreover, $\mathbf{1} \mathbf{a b}$ and $\mathbf{1 b c}$ show no cytotoxicity towards the human mammary epithelial cells MCF-10A and embryonic kidney epithelial cells Hek-293, in opposition to TCC, which exhibits a marked cytotoxicity on the same cell lines and shows a good antitumor activity on a panel of cell lines tested.
\end{abstract}

Keywords: antibacterial activity; triclocarban; cytoxicity; diarylureas; small molecules; Staphylococcus aureus; Enterococcus faecalis

\section{Introduction}

The polychlorinated aromatic antimicrobials triclocarban (TCC) and triclosan (TCS, Figure 1) are organic compounds that have been widely used in a vast range of applications $[1,2]$. Triclocarban, $N$-(4-chlorophenyl)- $N^{\prime}$-(3,4-dichlorophenyl)urea, is a highly effective and broad spectrum antimicrobial that has been successfully used in personal care products for over 60 years [3]. It belongs to the privileged class of diarylureas, which have been recently extensively reviewed for their multiple actions, including the anticancer [4], and have also been proposed for a repositioning as antimicrobial agents and/or for the treatment of new pandemics, including COVID-19 [5,6]. It is predominantly found in household necessities and personal care products, especially dermal cleaning products such as antibacterial bar/liquid soap, body lotion, deodorants, aftershave soaps, hand sanitizers, toothpaste, handwash and mouthwash [7]. Its concentration in the products can be as high as $1.5 \%$ [8]. It has been used for a long time to prevent food spoilage and infections because it perturbs microbial fatty acid synthesis and membrane formation [9]. TCC can be dechlorinated to 4,4'-dichlorocarbanilide (DCC), 1-(3-chlorophenyl)-3-phenylurea (MCC) and carbanilide (NCC), or either biologically or abiotically into 4-chloroaniline (4-CA) 
(Figure 1) [10]. Although TCC has been widely used for over 50 years, only recently some concerns about its endocrine disruptive properties were raised [11,12]. As a halogenated hydrocarbon, TCC is hardly biodegradable, and many studies have reported the occurrence of TCC in wastewater effluent, surface water, biosolid, sediment and soil [13]. Risks of TCC in the environment, on plants and animals, humans and microorganisms have been recently extensively described [14]. TCC is ranked in the top 10 Contaminant of Emerging Concern (CEC) occurrence. In September 2016, the U.S. Food and Drug Administration banned its use in over-the-counter hand and body washes [15], but this compound still retained a large market demand [16]. More recent studies are devoted to the removal of TCC and its dechlorinated congeners from soil, given their toxicity [17]. Thus, the desirable properties of next-generation antimicrobials should include broad-spectrum action and high efficacy toward pathogens but low toxicity to humans and should pose no risk of bioaccumulation. Recently, several diarylureas analogues of TCC were reported as antibacterial [18] and antifungal agents [19] bearing pentafluorosulfanyl and trifluoromethylcoumarine groups, respectively. As small molecules, their design should take into account a good oral bioavailability, following the "Lipinski's rule of drug-likeness", also named "the Rule of 5" (Ro5) [20].<smiles>O=C(Nc1ccc(Cl)cc1)Nc1ccc(Cl)c(Cl)c1</smiles><smiles>Oc1cc(Cl)ccc1Oc1ccc(Cl)cc1Cl</smiles><smiles>O=C(Nc1ccc(Cl)cc1)Nc1ccc(Cl)cc1</smiles><smiles>O=C(Nc1ccccc1)Nc1ccc(Cl)cc1</smiles>
4,4'-dichlorocarbanilide (DCC)

1-(3-chlorophenyl)-3-phenylurea (MCC)<smiles>O=C(Nc1ccccc1)Nc1ccccc1</smiles>

carbanilide (NCC)<smiles>Nc1ccc(Cl)cc1</smiles>

Figure 1. Triclocarban (TCC), triclosan and TCC transformation products.

In this paper, we report the synthesis of a series of diarylureas, particularly diphenylureas or bis-phenylureas (Figure 2), inspired by TCC, bearing different aryl moieties that we have previously studied, such as 2,6-xylyl, $p$-methyl, 3-4-difluoro and others [21-23], and their antimicrobial activity evaluation against Gram-positive and Gram-negative bacteria. Moreover, the anticancer effect of TCC (1ce) and its active analogues (1ab and $\mathbf{1 b c})$ was evaluated against several human cancer cell lines (breast cancer cells: MCF-7 and MDA-MB-231; uterine cancer cells: HeLa and Ishikawa; melanoma epithelial cells: A2058). Our outcomes clearly showed that TCC possesses a good antitumor activity against the panel of cell lines tested but, it is not selective because of its dramatic cytotoxic effects on normal human epithelial cells, MCF-10A, and embryonic kidney epithelial cells, Hek-293. Alternatively, both $\mathbf{1 a b}$ and $\mathbf{1 b c}$ do not possess any antitumor or cytotoxic activity, at least using the abovementioned cell lines and under our experimental conditions, but they exhibit an antibacterial activity comparable to or even higher than that of TCC. We are confident that these leads could represent promising tools for the development of new 
effective antibacterial agents and, whenever possible, also compounds with dual activity acting both as antibacterial and anticancer tools [24].<smiles>CCNC(=O)NCC</smiles>

$1 x y$ a:<smiles>Cc1ccc(I)cc1</smiles>

b:<smiles>Cc1cccc(C)c1I</smiles>

c:<smiles>Clc1ccc(I)cc1</smiles>

d:<smiles>COc1ccc(I)cc1</smiles>

e:<smiles>Clc1ccc(I)cc1Cl</smiles>

f:<smiles>[SiH3]c1ccccc1</smiles>

g:<smiles>Cc1ccccc1I</smiles>

h:<smiles>Clc1ccccc1I</smiles>

i:<smiles>Clc1ccccc1I</smiles>

j:<smiles>Fc1ccc(I)cc1I</smiles>

k:<smiles>Fc1cc(Cl)ccc1Cl</smiles>

I:<smiles>Fc1ccccc1I</smiles>

Figure 2. Diarylureas (a-1) under study.

\section{Results and Discussion}

\subsection{Chemistry}

Diphenylureas were easily prepared as depicted in Scheme 1. Final products were obtained by reacting the commercial anilines with the appropriate phenylisocyanate in acetone as reported in the literature [25].

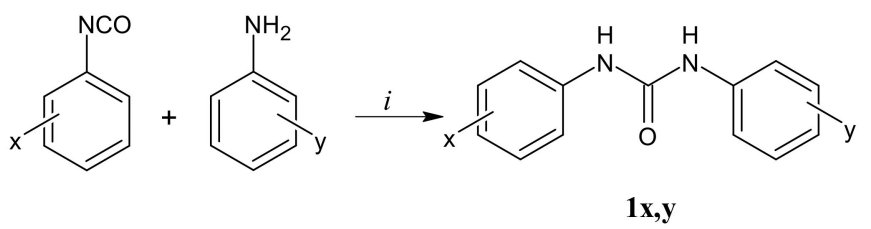

Scheme 1. Reagents and conditions: (i) acetone, $100{ }^{\circ} \mathrm{C}, 6 \mathrm{~h}$.

\subsection{Antibacterial Studies}

The in vitro Minimum Inhibitory Concentrations (MICs, $\mu \mathrm{g} / \mathrm{mL}$ ) were determined by the broth microdilution method according to the Clinical Laboratory Standards Institute (CLSI) guidelines. MIC values were recorded as the lowest concentration of compounds at which there was no optically detectable microorganism growth and evaluated by comparing the growth in each well visually with that of the growth in the control well for bacteria. All compounds were dissolved in dimethyl sulfoxide (DMSO) and were tested in a final concentration range of from 512 to $2 \mu \mathrm{g} / \mathrm{mL}$. MICs for the reference antibiotic norfloxacin against quality control strains were used to confirm the validity of the screen. According to the CLSI guidelines [26], diphenylureas were tested in vitro against a panel of Grampositive and Gram-negative bacteria belonging to the ATCC collection using the standard microdilution test for the determination of MICs. MICs of the synthesised compounds along with the parent compound TCC and with the standard antibiotic norfloxacin are listed in Table 1. The compounds were also analyzed in terms of molecular mass, $\log P$ (Table 1), number of hydrogen-bond donors and number of hydrogen-bond acceptors, and most of them obeyed Lipinski's rule [20]. Our results showed that the highest activity against $S$. aureus was obtained for compounds $\mathbf{1 a b}, \mathbf{1 b c}, \mathbf{1} \mathbf{b d}, \mathbf{1} \mathbf{f g}, \mathbf{1 b f}$ and $\mathbf{1 e f}$, showing MIC values of $16 \mu \mathrm{g} / \mathrm{mL}$, on a par with TCC. A slightly lower activity was observed for 1ad, 1be, 1gh and $\mathbf{1 g l}(\mathrm{MIC}=32 \mu \mathrm{g} / \mathrm{mL}$ ). The other compounds were less active or inactive. 
Based on our results, it seems that the 3,4-dichloro-disubstitution pattern, which is present in TCC, is not crucial for activity. Out of the six compounds bearing this substitution (TCC versus $\mathbf{1 b e}$, 1ef, 1eg, 1ae and 1de), only TCC showed high activity. Conversely, compounds $\mathbf{1 a b}, \mathbf{1 b c}, \mathbf{1 b d}$ and $\mathbf{1 b f}$, bearing a 2,6-xylyl, showed the highest antimicrobial activity against Staphylococcus aureus. Moreover, the activity against Enterococcus faecalis was interesting. E. faecalis is a ubiquitous member of the healthy human gut microbiota and also a common opportunistic pathogen and leading cause of nosocomial infections [27,28]. It has a high incidence for most infections, and the case numbers have been increasing in recent decades, likely due to its greater ability to persist in healthcare facilities and its higher abundance in the human gut microbiome [29]. Kim et al. (2016) [30] compared the bactericidal effects of plain and antibacterial soap containing $0.3 \%$ TCC. The authors found no significative difference between the effects of plain and medicated soap at either temperature, with the only exception of E. faecalis ATCC 19433 at $40^{\circ} \mathrm{C}$. 1ab and $\mathbf{1 b c}$ showed antibacterial activity higher than TCC (MIC $=32$ versus $64 \mu \mathrm{g} / \mathrm{mL}$ ) against this Gram-positive bacterium.

Table 1. Physicochemical properties and antibacterial activity results of diarylureas.

\begin{tabular}{|c|c|c|c|c|c|c|c|c|c|}
\hline \multirow[b]{3}{*}{ COMPD } & \multirow[b]{3}{*}{$\operatorname{cog} P^{a}$} & \multirow[b]{3}{*}{$\operatorname{milog} P^{b}$} & \multicolumn{7}{|c|}{ Microorganisms (MIC, $\mu \mathrm{g} / \mathrm{mL}$ ) } \\
\hline & & & \multicolumn{4}{|c|}{ Gram-Positive } & \multicolumn{3}{|c|}{ Gram-Negative } \\
\hline & & & S.a. 29213 & S.a. 6538 & S.a. $6538 \mathrm{P}$ & E.f. 29212 & $\begin{array}{c}\text { E.c. } \\
25922\end{array}$ & $\begin{array}{c}\text { P. } a . \\
27853\end{array}$ & $\begin{array}{r}\text { K.p. } \\
13883\end{array}$ \\
\hline $1 \mathrm{ab}$ & $4.24 \pm 0.30$ & 3.46 & $>256$ & 16 & 16 & 32 & $>256$ & $>256$ & $>256$ \\
\hline $1 b c$ & $4.77 \pm 0.33$ & 3.69 & 256 & 16 & 16 & 32 & $>256$ & $>256$ & $>256$ \\
\hline 1ad & $3.27 \pm 0.32$ & 3.65 & 128 & 32 & 32 & 128 & $>256$ & $>256$ & $>256$ \\
\hline $1 \mathrm{bd}$ & $3.73 \pm 0.32$ & 3.06 & $>256$ & 32 & 16 & 128 & $>256$ & $>256$ & $>256$ \\
\hline 1be & $5.68 \pm 0.36$ & 4.29 & 128 & 32 & 128 & 64 & $>256$ & $>256$ & $>256$ \\
\hline 1af & $3.32 \pm 0.29$ & 3.59 & 128 & 64 & 128 & 64 & $>256$ & $>256$ & $>256$ \\
\hline 1ag & $3.78 \pm 0.30$ & 3.99 & 256 & 128 & 256 & 128 & $>256$ & $>256$ & $>256$ \\
\hline 1ce (TCC) & $5.75 \pm 0.38$ & 5.11 & 16 & 128 & 16 & 64 & 256 & 256 & 256 \\
\hline 1ff (NCC) & $2.86 \pm 0.29$ & 3.14 & $>256$ & 128 & 64 & $>256$ & $>256$ & $>256$ & $>256$ \\
\hline 1fg & $3.32 \pm 0.29$ & 3.54 & 256 & 32 & 16 & 64 & $>256$ & $>256$ & 256 \\
\hline $1 \mathrm{bf}$ & $3.78 \pm 0.30$ & 3.01 & $>256$ & 16 & 16 & 64 & $>256$ & $>256$ & $>256$ \\
\hline $1 \mathrm{df}$ & $2.81 \pm 0.31$ & 3.20 & 256 & 64 & 128 & 128 & $>256$ & 256 & $>256$ \\
\hline 1gh & $3.83 \pm 0.32$ & 4.17 & 256 & 32 & 32 & 256 & $>256$ & $>256$ & $>256$ \\
\hline 1ef & $4.75 \pm 0.35$ & 4.43 & 256 & 16 & 128 & 128 & $>256$ & 256 & $>256$ \\
\hline 1cf (MCC) & $3.85 \pm 0.32$ & 3.82 & 128 & 64 & 128 & 64 & $>256$ & $>256$ & $>256$ \\
\hline $1 \mathrm{cc}(\mathrm{DCC})$ & $4.84 \pm 0.35$ & 4.50 & 256 & 128 & 256 & 128 & $>256$ & $>256$ & $>256$ \\
\hline 1 eg & $5.22 \pm 0.35$ & 4.83 & 256 & 64 & 128 & 64 & $>256$ & $>256$ & $>256$ \\
\hline $1 \mathrm{gj}$ & $4.16 \pm 0.52$ & 3.80 & $>256$ & 64 & 128 & 64 & $>256$ & $>256$ & $>256$ \\
\hline 1gk & $4.91 \pm 0.45$ & 4.31 & 256 & 128 & 64 & 64 & - & 256 & 256 \\
\hline $1 \mathrm{gl}$ & $3.28 \pm 0.40$ & 3.66 & 128 & 64 & 32 & 128 & $>256$ & $>256$ & $>256$ \\
\hline $\mathbf{1 f j}$ & $3.70 \pm 0.51$ & 3.40 & 256 & 128 & 64 & 128 & - & 256 & 256 \\
\hline 1ae & $5.22 \pm 0.35$ & 4.88 & 128 & 64 & 64 & 128 & $>256$ & $>256$ & $>256$ \\
\hline 1de & $4.70 \pm 0.37$ & 4.48 & 128 & 64 & 64 & 64 & $>256$ & $>256$ & $>256$ \\
\hline NRF & - & - & $0.5-2$ & - & - & $2-8$ & $0.03-0.12$ & $1-4$ & $0.5-1$ \\
\hline
\end{tabular}

Antibacterial activity was assessed using the CLSI assay (2012) [26]. Abbreviations: S.a.: S. aureus; E.f.: E. faecalis; E.c.: E. coli; P.a.: P. aeruginosa; K.p.: K. pneumoniae. NRF: norfloxacin; nt: not tested; ${ }^{a} \operatorname{cog} P$ values were computed with ACD Labs/Log P and ACD/ChemSketch 2012 from Advanced Chemistry Development (Toronto, Canada); ${ }^{\mathrm{b}}$ milog $P$ values were obtained from Molinspiration property calculation service FAQ, https: / / www.molinspiration.com/cgi-bin/properties (accessed on 2 February 2021); values according to CLSI (2012) [26].

\subsection{Cytotoxicity Studies}

In order to investigate the anticancer effects of compounds $\mathbf{1 a b}, \mathbf{1 b c}$ and $\mathbf{1 c e}$ (TCC), we used different human cancer cell models, namely, two breast cancer cells (MCF-7 and MDA-MB-231), two uterine cancer cells (HeLa and Ishikawa) and the melanoma epithelial cells A2058. Performing the MTT assay, we determined that compounds 1ab and 1bc did not elicit any anticancer effect towards all the cancer cell lines used, at least until the concentration of $200 \mu \mathrm{g} / \mathrm{mL}$ (Table 2). On the contrary, TCC, used as the reference molecule, 
exerted a strong anticancer effect against the cancer cell lines, with $\mathrm{IC}_{50}$ values between 0.64 and $1.68 \mu \mathrm{g} / \mathrm{mL}$. Unfortunately, TCC also exhibited a marked cytotoxicity on the normal cells used in this assay, the human mammary epithelial cells MCF-10A and embryonic kidney epithelial cells Hek-293, with $\mathrm{IC}_{50}$ values of $1.43 \pm 0.7$ and $1.60 \pm 0.8 \mu \mathrm{g} / \mathrm{mL}$, respectively. Instead, compounds $\mathbf{1} \mathbf{a b}$ and $\mathbf{1 b c}$ did not possess any cytotoxicity towards the same normal cancer cell lines at least below the concentration of $200 \mu \mathrm{g} / \mathrm{mL}$.

Table 2. $\mathrm{IC}_{50}$ values $(\mu \mathrm{g} / \mathrm{mL})$ of compounds $\mathbf{1 a b}, \mathbf{1} \mathbf{b c}$ and TCC on human breast, uterine and melanoma cancer cells as determined by using the MTT assay. $\mathrm{IC}_{50}$ values are the mean $\pm \mathrm{SD}$ of three independent experiments performed in triplicate.

\begin{tabular}{llll}
\hline Cell Lines & \multicolumn{1}{l}{ IC $_{\mathbf{5 0}}(\boldsymbol{\mu g} / \mathbf{m L})$} & \\
\hline & 1ab & 1bc & 1ce (TCC) \\
\hline MDA-MB-231 & $>200$ & $>200$ & $0.89 \pm 0.8$ \\
MCF-7 & $>200$ & $>200$ & $0.64 \pm 0.5$ \\
HeLa & $>200$ & $>200$ & $1.01 \pm 1.1$ \\
Ishikawa & $>200$ & $>200$ & $1.68 \pm 0.9$ \\
A2058 & $>200$ & $>200$ & $0.89 \pm 0.7$ \\
MCF-10A & $>200$ & $>200$ & $1.43 \pm 0.7$ \\
Hek-293 & $>200$ & $>200$ & $1.60 \pm 0.8$ \\
\hline
\end{tabular}

\section{Materials and Methods}

\subsection{Chemistry}

Chemicals were purchased from Sigma-Aldrich or Lancaster. Yields refer to purified products and were not optimized. Compound stuctures were confirmed by ordinary spectrometric analyses ( ${ }^{1} \mathrm{H}$ NMR, ${ }^{13} \mathrm{C}$ NMR, LC-MS, IR, Elemental Analysis). Melting points were determined on a Gallenkamp melting point apparatus in open glass capillary tubes and are uncorrected. ${ }^{1} \mathrm{H}$ and ${ }^{13} \mathrm{C}$ NMR spectra were registered on a Varian VX Mercury spectrometer operating at 300 and $75 \mathrm{MHz}$ for ${ }^{1} \mathrm{H}$ and ${ }^{13} \mathrm{C}$, respectively, or an Agilent $500 \mathrm{MHz}$ operating at 500 and $125 \mathrm{MHz}$ for ${ }^{1} \mathrm{H}$ and ${ }^{13} \mathrm{C}$, respectively, using DMSO$d_{6}$ as solvent. Chemical shifts are reported in parts per million (ppm) relative to solvent resonance: $\delta=2.48 \mathrm{ppm}\left({ }^{1} \mathrm{H} \mathrm{NMR}\right)$ and $\delta=39.9 \mathrm{ppm}\left({ }^{13} \mathrm{C} \mathrm{NMR}\right)$. $J$ values are given in $\mathrm{Hz}$. Abbreviations "s-singlet, d-doublet, t-triplet, m-multiplet" are used. Gas chromatography (GC)/mass spectroscopy (MS) was performed on a Hewlett-Packard 6890-5973 MSD at low resolution. Liquid chromatography (LC)/mass spectroscopy (MS) was performed on a spectrometer Agilent 1100 series LC-MSD Trap System VL. The molecular ion was designed as " $\mathrm{M}^{+\prime}$. Elemental analyses were performed on a Eurovector Euro EA 3000 analyzer. The data for $\mathrm{C}, \mathrm{H}, \mathrm{N}$ were within \pm 0.4 of theoretical values.

1-(2,6-Dimethylphenyl)-3-p-tolylurea (1ab) [31]. A solution of 2,6-dimethylphenyl isocyanate $(0.26 \mathrm{~mL}, 1.87 \mathrm{mmol})$ in acetone $\mathrm{RP}(4 \mathrm{~mL})$ was added in three portions (in $1.5 \mathrm{~h})$ to a solution of $p$-toluidine $(0.20 \mathrm{~g}, 1.87 \mathrm{mmol})$ in acetone $\mathrm{RP}(4 \mathrm{~mL})$. The resulting mixture was refluxed for $6 \mathrm{~h}$. The solid was filtered to give $1 \mathrm{ab}$ as a white solid $(0.41 \mathrm{~g}, 86 \%)$, which was recrystallized from $\mathrm{CHCl}_{3}$ giving $0.35 \mathrm{~g}(73 \%)$ of white crystals: mp $242-243{ }^{\circ} \mathrm{C} ; \mathrm{GC} / \mathrm{MS}$ $(70 \mathrm{eV}) \mathrm{m} / \mathrm{z}(\%): 254\left(\mathrm{M}^{+}, 24\right), 107(100) ; \mathrm{IR}(\mathrm{KBr}): 3288(\mathrm{NH}), 1642(\mathrm{C}=\mathrm{O}) \mathrm{cm}^{-1}{ }^{1}{ }^{1} \mathrm{H}$ NMR $\left(300 \mathrm{MHz}, \mathrm{DMSO}-d_{6}\right): \delta 2.18\left(\mathrm{~s}, 6 \mathrm{H}, 2 \mathrm{CH}_{3}\right), 2.21\left(\mathrm{~s}, 3 \mathrm{H}, \mathrm{CH}_{3}\right), 6.98-7.10(\mathrm{~m}, 5 \mathrm{H}, \mathrm{Ar}), 7.31(\mathrm{~d}$, $J=8.2 \mathrm{~Hz}, 2 \mathrm{H}, \mathrm{Ar}), 7.63$ (br s, $1 \mathrm{H}, \mathrm{NH}$, exch. $\left.\mathrm{D}_{2} \mathrm{O}\right), 8.57$ (br s, $1 \mathrm{H}, \mathrm{NH}$, exch. $\mathrm{D}_{2} \mathrm{O}$ ); ${ }^{13} \mathrm{C}$ NMR (75 MHz, DMSO- $\left.d_{6}\right): \delta 18.7$ (2C), 20.8 (1C), 118.5 (2C), 126.3 (1C), $128.1(2 \mathrm{C}), 129.5$ (2C), 130.6 (1C), 135.9 (2C), 136.0 (1C), 138.2 (1C), 153.6 (1C). Anal. calcd for $\mathrm{C}_{16} \mathrm{H}_{18} \mathrm{~N}_{2} \mathrm{O} \cdot 0.20$ $\mathrm{H}_{2} \mathrm{O}(257.75) \%$ : C 74.51; H 7.19; N 10.86. Found \%: C 74.76; H 7.00; N 10.85.

1-(2,6-Dimethylphenyl)-3-(4-chlorophenyl)urea (1bc) [32]. Prepared from 4-CA and 2,6dimethylphenyl isocyanate. Yield: $72 \%$ (needle-like white crystals): $\mathrm{mp} 244-245{ }^{\circ} \mathrm{C}$ $\left(\mathrm{CHCl}_{3}\right) ; \mathrm{GC} / \mathrm{MS}(70 \mathrm{eV}) \mathrm{m} / z(\%): 274\left(\mathrm{M}^{+}, 13\right), 127$ (100); IR (KBr): $3282(\mathrm{NH}), 1643$ $(\mathrm{C}=\mathrm{O}) \mathrm{cm}^{-1} ;{ }^{1} \mathrm{H}$ NMR $\left(300 \mathrm{MHz}, \mathrm{DMSO}-d_{6}\right): \delta 2.18\left(\mathrm{~s}, 6 \mathrm{H}, 2 \mathrm{CH}_{3}\right), 7.02-7.08(\mathrm{~m}, 3 \mathrm{H}, \mathrm{Ar})$, $7.27(\mathrm{~d}, J=8.8 \mathrm{~Hz}, 2 \mathrm{H}, \mathrm{Ar}), 7.47(\mathrm{~d}, J=8.8 \mathrm{~Hz}, 2 \mathrm{H}, \mathrm{Ar}) 7.73$ (br s, 1H, NH, exch. $\mathrm{D}_{2} \mathrm{O}$ ), 8.86 (br s, $1 \mathrm{H}, \mathrm{NH}$, exch. $\left.\mathrm{D}_{2} \mathrm{O}\right) ;{ }^{13} \mathrm{C}$ NMR (125 MHz, DMSO-d 6 ): $\delta 18.7$ (2C), $119.8(2 \mathrm{C})$, 
125.3 (1C), 126.5 (1C), 128.2 (2C), 129.0 (2C), 135.6 (1C), 136.0 (2C), 139.8 (1C), 153.5 (1C). Anal. calcd for $\mathrm{C}_{15} \mathrm{H}_{15} \mathrm{ClN}_{2} \mathrm{O}$ (274.59)\%: C 65.57; H 5.50; N 10.20. Found\%: C 65.59; H 5.65; $\mathrm{N} 10.59$.

1-(4-Methoxyphenyl)-3-(p-tolyl)urea (1ad) [33]. Prepared from $p$-toluidine and $p$-methoxyphenyl isocyanate. Yield: $65 \%$ (needle-like white crystals): $\mathrm{mp} 241-242{ }^{\circ} \mathrm{C}(\mathrm{EtOH} 96 \%)$; LC/MS (70 eV) m/z (\%): 276 [M $\left.{ }^{+},+23\right]$; IR (KBr): $3283(\mathrm{NH}), 1644(\mathrm{C}=\mathrm{O}) \mathrm{cm}^{-1}$; ${ }^{1} \mathrm{H}$ NMR $\left(300 \mathrm{MHz}, \mathrm{DMSO}-d_{6}\right): \delta 2.21\left(\mathrm{~s}, 3 \mathrm{H}, \mathrm{CH}_{3}\right), 3.68\left(\mathrm{~s}, 3 \mathrm{H}, \mathrm{CH}_{3}\right), 6.84(\mathrm{~d}, J=8.8 \mathrm{~Hz}, 2 \mathrm{H}, \mathrm{Ar})$, $7.04(\mathrm{~d}, J=8.2 \mathrm{~Hz}, 2 \mathrm{H}, \mathrm{Ar}), 7.26-7.36$ (m, 4H, Ar), 8.38 (br s, 1H, NH, exch. $\left.\mathrm{D}_{2} \mathrm{O}\right), 8.43$ (br s, $1 \mathrm{H}, \mathrm{NH}$, exch. $\left.\mathrm{D}_{2} \mathrm{O}\right) ;{ }^{13} \mathrm{C}$ NMR $\left(75 \mathrm{MHz}, \mathrm{DMSO}-d_{6}\right): \delta 20.8(1 \mathrm{C}), 55.6(1 \mathrm{C}), 114.4(2 \mathrm{C})$, 118.6 (2C), 120.4 (2C), 129.6 (2C), 130.8 (1C), 133.3 (1C), 137.7 (1C), 153.2 (1C), 154.9 (1C). Anal. calcd for $\mathrm{C}_{15} \mathrm{H}_{16} \mathrm{~N}_{2} \mathrm{O}_{2} \cdot 0.33 \mathrm{H}_{2} \mathrm{O}(262.12) \%$ : C 68.68; $\mathrm{H}$ 6.40; $\mathrm{N} 10.68$. Found \%: C 68.37; H 6.16; N 10.55 .

1-(4-Methoxyphenyl)-3-(2,6-dimethylphenyl)urea (1bd) [32]. Prepared from $p$-methoxyphenyl isocyanate and 2,6-dimethylaniline. Yield: $66 \%$ (needle-like white crystals): mp $225-226{ }^{\circ} \mathrm{C}$ $\left(\mathrm{CHCl}_{3}\right)$; LC/MS (70 eV) m/z (\%): 293 [M+, +23]; IR (KBr): $3262(\mathrm{NH}), 1643(\mathrm{C}=\mathrm{O}) \mathrm{cm}^{-1}$; ${ }^{1} \mathrm{H}$ NMR $\left(300 \mathrm{MHz}, \mathrm{DMSO}-d_{6}\right): \delta 2.18\left(\mathrm{~s}, 6 \mathrm{H}, 2 \mathrm{CH}_{3}\right), 3.68\left(\mathrm{~s}, 3 \mathrm{H}, \mathrm{CH}_{3}\right), 6.82(\mathrm{~d}, \mathrm{~J}=8.8 \mathrm{~Hz}$, 2H, Ar), 7.03 (s, 2H, Ar), 7.33 (d, J = 8.8 Hz, 2H, Ar), 7.59 (s, 1H, Ar) 8.37 (br s, 1H, NH, exch. $\left.\mathrm{D}_{2} \mathrm{O}\right), 8.50$ (br s, $1 \mathrm{H}, \mathrm{NH}$, exch. $\left.\mathrm{D}_{2} \mathrm{O}\right) ;{ }^{13} \mathrm{C}$ NMR $\left(75 \mathrm{MHz}, \mathrm{DMSO}-d_{6}\right): \delta 18.7(2 \mathrm{C})$, 55.6 (1C), $114.4(2 \mathrm{C}), 120.1$ (2C), 120.3 (2C), 126.2 (1C), 128.1 (2C), 133.9 (1C), 135.9 (1C), 153.8 (1C), 154.6 (1C). Anal. calcd for $\mathrm{C}_{16} \mathrm{H}_{18} \mathrm{~N}_{2} \mathrm{O}_{2}(270.33) \%$ : C 71.09; $\mathrm{H}$ 6.71; N 10.36. Found \%: C 70.70; H 6.58; N 10.35.

1-(2,6-Dimethylphenyl)-3-(3,4-dichlorophenyl)urea (1be). Prepared from 2,6-dimethylaniline and 3,4-dichlorophenyl isocyanate. Yield: 76\% (white crystals): $\mathrm{mp} 249-250{ }^{\circ} \mathrm{C}\left(\mathrm{CHCl}_{3} /\right.$ $\mathrm{MeOH} /$ Acetone); LC/MS (70 eV) m/z (\%): $331\left[\mathrm{M}^{+},+23\right]$; IR (KBr): $3301(\mathrm{NH}), 1639$ $(\mathrm{C}=\mathrm{O}) \mathrm{cm}^{-1} ;{ }^{1} \mathrm{H}$ NMR (300 MHz, DMSO-d $): \delta 2.17\left(\mathrm{~s}, 6 \mathrm{H}, 2 \mathrm{CH}_{3}\right), 7.05(\mathrm{~s}, 3 \mathrm{H}, \mathrm{Ar}), 7.31$ $(\mathrm{d}, J=8.8 \mathrm{~Hz}, 1 \mathrm{H}, \mathrm{Ar}), 7.46(\mathrm{~d}, J=8.8 \mathrm{~Hz}, 1 \mathrm{H}, \mathrm{Ar}), 7.84$ (br s, 2H, Ar + NH, exch. $\left.\mathrm{D}_{2} \mathrm{O}\right)$, 9.06 (br s, $1 \mathrm{H}, \mathrm{NH}$, exch. $\left.\mathrm{D}_{2} \mathrm{O}\right) ;{ }^{13} \mathrm{C} \mathrm{NMR}\left(75 \mathrm{MHz}, \mathrm{DMSO}-d_{6}\right): \delta 18.6(2 \mathrm{C}), 118.5(1 \mathrm{C})$, 119.5 (1C), 123.0 (1C), 126.7 (1C), 128.2 (2C), 130.9 (1C), $131.4(1 C), 135.3(1 C), 136.1(2 C)$, 141.0 (1C), 153.4 (1C). Anal. calcd for $\mathrm{C}_{15} \mathrm{H}_{14} \mathrm{Cl}_{2} \mathrm{~N}_{2} \mathrm{O} \cdot 0.50 \mathrm{H}_{2} \mathrm{O}(317.05) \%$ : C 56.62; $\mathrm{H} 4.75$; N 8.80. Found\%: C 56.78; H 4.43; N 8.93.

1-(4-Methylphenyl)-3-phenylurea (1af) [34]. Prepared from $p$-toluidine and phenyl isocyanate. Yield: 80\% (needle-like white crystals): $\mathrm{mp} 240-242{ }^{\circ} \mathrm{C}\left(\mathrm{CHCl}_{3} /\right.$ acetone); LC/MS (70 eV) m/z (\%): $249\left[\mathrm{M}^{+},+23\right]$; IR (KBr): $3301(\mathrm{NH}), 1634(\mathrm{C}=\mathrm{O}) \mathrm{cm}^{-1} ;{ }^{1} \mathrm{H}$ NMR $(500 \mathrm{MHz}$, DMSO-d $\left.{ }_{6}\right): \delta 2.22\left(\mathrm{~s}, 3 \mathrm{H}, \mathrm{CH}_{3}\right) 6.93(\mathrm{t}, J=7.3 \mathrm{~Hz}, 1 \mathrm{H}, \mathrm{Ar}), 7.06(\mathrm{~d}, J=8.3 \mathrm{~Hz}, 2 \mathrm{H}, \mathrm{Ar}), 7.25$ $(\mathrm{t}, J=7.5 \mathrm{~Hz}, 2 \mathrm{H}, \mathrm{Ar}), 7.31(\mathrm{~d}, J=8.3 \mathrm{~Hz}, 2 \mathrm{H}, \mathrm{Ar}), 7.42(\mathrm{~d}, J=8.3 \mathrm{~Hz}, 2 \mathrm{H}, \mathrm{Ar}), 8.51(\mathrm{~s}, 1 \mathrm{H}$, $\mathrm{NH}), 8.57$ (s, 1H, NH); ${ }^{13} \mathrm{C}$ NMR (125 MHz, DMSO-d $)$ : $\delta 20.8$ (1C), $118.6(2 \mathrm{C}), 118.7$ (2C), $122.1(1 \mathrm{C}), 129.2$ (2C), 129.6 (2C), 131.0 (1C), $137.6(1 \mathrm{C}), 140.2$ (1C), 153.0 (1C).

1-(2-Methylphenyl)-3-(4-methylphenyl)urea (1ag) [35]. Prepared from $p$-toluidine and o-methylphenyl isocyanate. Yield: 74\% (white solid): $\mathrm{mp} 241-242{ }^{\circ} \mathrm{C}$; LC/MS (70 eV) $\mathrm{m} / \mathrm{z}$ (\%): 263 [M $\left.{ }^{+},+23\right]$; IR (KBr): 3305 (NH), 1643 (C=O) cm ${ }^{-1} ;{ }^{1} \mathrm{H}$ NMR (300 MHz, DMSO- $\left.d_{6}\right)$ : $\delta 2.21\left(\mathrm{~s}, 6 \mathrm{H}, 2 \mathrm{CH}_{3}\right), 6.91(\mathrm{t}, J=7.6 \mathrm{~Hz}, 1 \mathrm{H}, \mathrm{Ar}), 7.05-7.15(\mathrm{~m}, 4 \mathrm{H}, \mathrm{Ar}), 7.32(\mathrm{~d}, J=8.2 \mathrm{~Hz}$, $2 \mathrm{H}, \mathrm{Ar}$ ), 7.82 (d overlapping $\mathrm{s}$ at $7.83 \mathrm{ppm}, J=8.8 \mathrm{~Hz}, 1 \mathrm{H}, \mathrm{Ar}$ ), 7.83 (s overlapping d at 7.82 ppm, $1 \mathrm{H}, \mathrm{NH}$, exch. $\left.\mathrm{D}_{2} \mathrm{O}\right), 8.88\left(\mathrm{~s}, 1 \mathrm{H}, \mathrm{NH}\right.$, exch. $\left.\mathrm{D}_{2} \mathrm{O}\right) ;{ }^{13} \mathrm{C}$ NMR $\left(75 \mathrm{MHz}, \mathrm{DMSO}-d_{6}\right)$ : $\delta 18.3(1 \mathrm{C}), 20.8(1 \mathrm{C}), 118.5(2 \mathrm{C}), 121.4(1 \mathrm{C}), 122.9(1 \mathrm{C}), 126.6(1 \mathrm{C}), 127.8(1 \mathrm{C}), 129.6(2 \mathrm{C})$, $130.6(1 \mathrm{C}), 130.9(1 \mathrm{C}), 137.7(1 \mathrm{C}), 137.9(1 \mathrm{C}), 153.1(1 \mathrm{C})$. Anal. calcd for $\mathrm{C}_{15} \mathrm{H}_{16} \mathrm{~N}_{2} \mathrm{O} \cdot 0.14 \mathrm{H}_{2} \mathrm{O}$ (242.87) \%: C 74.18; H 6.76; N 11.53. Found\%: C 74.52; H 6.56; N 11.61.

1-(3,4-Dichlorophenyl)-3-(4-chlorophenyl)urea (1ce, TCC) [36]. Prepared from 3,4-dichloroaniline and $p$-chlorophenyl isocyanate. Yield: $29 \%$ (white solid): $\mathrm{mp} \geq 250{ }^{\circ} \mathrm{C}$; LC/MS $(70 \mathrm{eV}) \mathrm{m} / \mathrm{z}(\%): 317$ [M+]; IR (KBr): $3296(\mathrm{NH}), 1639(\mathrm{C}=\mathrm{O}) \mathrm{cm}^{-1} ;{ }^{1} \mathrm{H}$ NMR $(500 \mathrm{MHz}$, DMSO- $\left.d_{6}\right): \delta 7.31(\mathrm{~d}, J=8.8 \mathrm{~Hz}, 3 \mathrm{H}, \mathrm{Ar}), 7.45-7.50(\mathrm{~m}, 3 \mathrm{H}, \mathrm{Ar}), 7.84(\mathrm{~d}, J=2.4 \mathrm{~Hz}, 1 \mathrm{H}, \mathrm{Ar})$, 8.92 (s, 1H, NH, exch. $\left.\mathrm{D}_{2} \mathrm{O}\right), 8.99$ (s, 1H, NH, exch. $\left.\mathrm{D}_{2} \mathrm{O}\right) ;{ }^{13} \mathrm{C}$ NMR $\left(125 \mathrm{MHz}, \mathrm{DMSO}-d_{6}\right)$ : $\delta 118.9$ (1C), $119.8(1 \mathrm{C}), 120.5$ (1C), 123.7 (2C), 126.2 (1C), 129.1 (2C), $131.0(1 \mathrm{C}), 131.5(1 \mathrm{C})$, $138.7(1 \mathrm{C}), 140.3(1 \mathrm{C}), 152.7(1 \mathrm{C})$. Anal. calcd for $\mathrm{C}_{13} \mathrm{H}_{9} \mathrm{Cl}_{3} \mathrm{~N}_{2} \mathrm{O}$ (315.58) \%: C 49.48; $\mathrm{H}$ 2.87; N 8.88. Found\%: C 49.48; H 3.02; N 8.79. 
1,3-Diphenylurea (1ff, NCC) [37]. Prepared from aniline and phenyl isocyanate. Yield: 77\% (white solid): $\mathrm{mp} 242-243{ }^{\circ} \mathrm{C}$; LC/MS (70 eV) $m / z(\%): 235\left[\mathrm{M}^{+},+23\right]$; IR (KBr): $3328(\mathrm{NH}), 1647(\mathrm{C}=\mathrm{O}) \mathrm{cm}^{-1}{ }^{1} \mathrm{H}$ NMR $\left(300 \mathrm{MHz}, \mathrm{DMSO}-d_{6}\right): \delta 6.95(\mathrm{t}, J=7.3 \mathrm{~Hz}, 2 \mathrm{H}, \mathrm{Ar})$, $7.26(\mathrm{t}, J=7.9 \mathrm{~Hz}, 4 \mathrm{H}, \mathrm{Ar}) 7.43(\mathrm{~d}, J=7.6 \mathrm{~Hz}, 4 \mathrm{H}, \mathrm{Ar}), 8.65\left(\mathrm{~s}, 2 \mathrm{H}, \mathrm{NH}\right.$, exch. $\left.\mathrm{D}_{2} \mathrm{O}\right) ;{ }^{13} \mathrm{C} \mathrm{NMR}$ (75 MHz, DMSO- $\left.d_{6}\right): \delta 118.6(4 \mathrm{C}), 122.3(2 \mathrm{C}), 129.2(4 \mathrm{C}), 140.1(2 \mathrm{C}), 152.9$ (1C). Anal. calcd for $\mathrm{C}_{13} \mathrm{H}_{12} \mathrm{~N}_{2} \mathrm{O}(212,24) \%$ : C 73.56; H 5.70; $\mathrm{N}$ 13.20. Found\%: C 73.63; H 5.61; N 13.19.

1-(2-Methylphenyl)-3-phenylurea (1fg) [33]. Prepared from aniline and o-tolyl isocyanate. Yield: $77 \%$ (white solid): $\mathrm{mp} 241-242{ }^{\circ} \mathrm{C}$; LC/MS (70 eV) $\mathrm{m} / z(\%): 249\left[\mathrm{M}^{+},+23\right]$; IR (KBr): $3269(\mathrm{NH}), 1631(\mathrm{C}=\mathrm{O}) \mathrm{cm}^{-1} ;{ }^{1} \mathrm{H}$ NMR (300 MHz, DMSO- $\left.d_{6}\right): \delta 2.21\left(\mathrm{~s}, 3 \mathrm{H}, \mathrm{CH}_{3}\right), 6.89-6.96$ $(\mathrm{m}, 2 \mathrm{H}, \mathrm{Ar}), 7.09-7.16(\mathrm{~m}, 2 \mathrm{H}, \mathrm{Ar}), 7.26(\mathrm{t}, J=7.6 \mathrm{~Hz}, 2 \mathrm{H}, \mathrm{Ar}), 7.44(\mathrm{~d}, J=7.6 \mathrm{~Hz}, 2 \mathrm{H}, \mathrm{Ar})$, $7.81(\mathrm{~d}, J=8.2 \mathrm{~Hz}, 1 \mathrm{H}, \mathrm{Ar}), 7.90\left(\mathrm{~s}, 1 \mathrm{H}, \mathrm{NH}\right.$, exch. $\left.\mathrm{D}_{2} \mathrm{O}\right), 9.01\left(\mathrm{~s}, 1 \mathrm{H}, \mathrm{NH}\right.$, exch. $\left.\mathrm{D}_{2} \mathrm{O}\right)$; ${ }^{13} \mathrm{C}$ NMR (75 MHz, DMSO- $\left.d_{6}\right): \delta 18.3$ (1C), $118.4(2 \mathrm{C}), 121.5$ (1C), $122.1(1 \mathrm{C}), 123.1(1 \mathrm{C})$, 126.6 (1C), 128.0 (1C), 129.3 (2C), 130.6 (1C), 137.8 (1C), 140.3 (1C), 153.1 (1C). Anal. calcd for $\mathrm{C}_{14} \mathrm{H}_{14} \mathrm{~N}_{2} \mathrm{O} \cdot 0.16 \mathrm{H}_{2} \mathrm{O}(229.27) \%$ : C 73.34; $\mathrm{H}$ 6.30; $\mathrm{N}$ 12.22. Found\%: $\mathrm{C} 73.65 ; \mathrm{H} 6.08$; N 12.27.

1-(2,6-Dimethylphenyl)-3-phenylurea (1bf) [32]. Prepared from 2,6-dimethylaniline and phenyl isocyanate. Yield: quantitative (white solid): $\mathrm{mp} 240-241^{\circ} \mathrm{C}$; LC/MS $(70 \mathrm{eV}) \mathrm{m} / \mathrm{z}$ (\%): 263 [M $\left.\mathrm{M}^{+},+23\right]$; IR (KBr): $3287(\mathrm{NH}), 1633(\mathrm{C}=\mathrm{O}) \mathrm{cm}^{-1}$; ${ }^{1} \mathrm{H}$ NMR $(300 \mathrm{MHz}, \mathrm{DMSO}-$ $\left.d_{6}\right): \delta 2.18\left(\mathrm{~s}, 6 \mathrm{H}, 2 \mathrm{CH}_{3}\right), 6.91(\mathrm{~d}, J=7.0 \mathrm{~Hz}, 1 \mathrm{H}, \mathrm{Ar}), 7.04(\mathrm{~s}, 3 \mathrm{H}, \mathrm{Ar}), 7.23(\mathrm{t}, J=7.9 \mathrm{~Hz}$, $2 \mathrm{H}, \mathrm{Ar}), 7.43(\mathrm{~d}, J=7.6 \mathrm{~Hz}, 2 \mathrm{H}, \mathrm{Ar}), 7.69\left(\mathrm{~s}, 1 \mathrm{H}, \mathrm{NH}\right.$, exch. $\left.\mathrm{D}_{2} \mathrm{O}\right), 8.72(\mathrm{~s}, 1 \mathrm{H}, \mathrm{NH}$, exch. $\left.\mathrm{D}_{2} \mathrm{O}\right) ;{ }^{13} \mathrm{C}$ NMR $\left(75 \mathrm{MHz}\right.$, DMSO- $\left.d_{6}\right): \delta 18.7(2 \mathrm{C}), 118.3(2 \mathrm{C}), 121.8(2 \mathrm{C}), 126.4(1 \mathrm{C}), 128.1$ (2C), 129.1 (2C), $135.8(1 \mathrm{C}), 136.0$ (1C), 140.8 (1C), 153.6 (1C). Anal. calcd for $\mathrm{C}_{15} \mathrm{H}_{16} \mathrm{~N}_{2} \mathrm{O}$ (240.12)\%: C 74.97; H 6.71; N 11.66. Found\%: C 75.41; H 6.60; N 11.80.

1-(4-Methoxyphenyl)-3-phenylurea (1df) [38]. Prepared from phenyl isocyanate and $p$-anisidine. Yield: $64 \%$ (white needle-shaped fine crystals): mp $216-218{ }^{\circ} \mathrm{C} ; \mathrm{LC} / \mathrm{MS}(70 \mathrm{eV})$ $m / z(\%): 265\left[\mathrm{M}^{+},+23\right]$; IR (KBr): $3288(\mathrm{NH}), 1633(\mathrm{C}=\mathrm{O}) \mathrm{cm}^{-1} ;{ }^{1} \mathrm{H}$ NMR $(300 \mathrm{MHz}$, DMSO- $\left.d_{6}\right): \delta 3.69\left(\mathrm{~s}, 3 \mathrm{H}, \mathrm{O}-\mathrm{CH}_{3}\right), 6.85(\mathrm{~d}, J=8.8 \mathrm{~Hz}, 2 \mathrm{H}, \mathrm{Ar}), 6.93(\mathrm{t}, J=7.0 \mathrm{~Hz}, 1 \mathrm{H}, \mathrm{Ar})$, $7.24(\mathrm{t}, J=7.6 \mathrm{~Hz}, 2 \mathrm{H}, \mathrm{Ar}), 7.34(\mathrm{~d}, J=8.8 \mathrm{~Hz}, 2 \mathrm{H}, \mathrm{Ar}), 7.42(\mathrm{~d}, J=8.2 \mathrm{~Hz}, 2 \mathrm{H}, \mathrm{Ar}), 8.44(\mathrm{~s}$, $1 \mathrm{H}, \mathrm{NH}$, exch. $\left.\mathrm{D}_{2} \mathrm{O}\right), 8.55\left(\mathrm{~s}, 1 \mathrm{H}, \mathrm{NH}\right.$, exch. $\left.\mathrm{D}_{2} \mathrm{O}\right) ;{ }^{13} \mathrm{C}$ NMR $\left(125 \mathrm{MHz}\right.$, DMSO- $\left.d_{6}\right): \delta 55.6$ (1C), 114.4 (2C), 118.5 (2C), 120.4 (2C), 122 (1C), 129.2 (2C), 133.2 (1C), 140.3 (1C), 153.2 (1C), 154.9 (1C). Anal. calcd for $\mathrm{C}_{14} \mathrm{H}_{14} \mathrm{~N}_{2} \mathrm{O}_{2} \cdot 0.30 \mathrm{H}_{2} \mathrm{O}(248.27) \%$ : C 67.73; H 5.95; N 11.28. Found \%: C 67.43; H 5.76; N 11.14.

1-(2-Chlorophenyl)-3-(2-methylphenyl)urea (1gh). Prepared from o-chloroaniline and otolyl isocyanate. Yield: $87 \%$ (off-white crystals): $\mathrm{mp} 223-224{ }^{\circ} \mathrm{C}$ (acetone $/ \mathrm{CHCl}_{3}$ ); LC/MS $(70 \mathrm{eV}) \mathrm{m} / z(\%): 283\left[\mathrm{M}^{+},+23\right]$; IR (KBr): $3284(\mathrm{NH}), 1643(\mathrm{C}=\mathrm{O}) \mathrm{cm}^{-1} ;{ }^{1} \mathrm{H}$ NMR $(300 \mathrm{MHz}$, DMSO- $\left.d_{6}\right): \delta 3.33\left(\mathrm{~s}, 3 \mathrm{H}, \mathrm{CH}_{3}\right), 6.96-7.03(\mathrm{~m}, 2 \mathrm{H}, \mathrm{Ar}), 7.11-7.18(\mathrm{~m}, 2 \mathrm{H}, \mathrm{Ar}), 7.24-7.30$ $(\mathrm{m}, 1 \mathrm{H}, \mathrm{Ar}), 7.44(\mathrm{q}, J=3.2 \mathrm{~Hz}, 1 \mathrm{H}, \mathrm{Ar}), 7.75(\mathrm{~d}, J=8.2 \mathrm{~Hz}, 1 \mathrm{H}, \mathrm{Ar}), 8.11(\mathrm{~d}, J=1.7 \mathrm{~Hz}$, $1 \mathrm{H}, \mathrm{Ar}), 8.63\left(\mathrm{~d}, J=12.4 \mathrm{~Hz}, 2 \mathrm{H}, \mathrm{NH}\right.$ exch. $\left.\mathrm{D}_{2} \mathrm{O}\right) ;{ }^{13} \mathrm{C}$ NMR $\left(125 \mathrm{MHz}\right.$, DMSO- $\left.d_{6}\right): 18.3$ (1C), 121.1 (1C), 122.2 (1C), 123.4 (1C), 123.5 (1C), 126.3 (2C), 127.7 (1C), 128.5 (1C), 129.4 (1C), 130.5 (1C), 136.4 (1C), 137.2 (1C), 152.7 (1C). Anal. calcd for $\mathrm{C}_{14} \mathrm{H}_{13} \mathrm{~N}_{2} \mathrm{OCl}(260.07) \%$ : C 64.49; H 5.03; N 10.74. Found\%: C 64.20; H 4.97; N 10.68.

1-(3,4-Dichlorophenyl)-3-phenylurea (1ef) [39]. Prepared from 3,4-dichloroaniline and phenyl isocyanate. Yield: $76 \%$ (slightly greenish crystals): $\mathrm{mp} 215-216^{\circ} \mathrm{C}$ (acetone $/ \mathrm{CHCl}_{3}$ ); LC/MS (70 eV) $m / z(\%): 280\left[\mathrm{M}^{+},-1\right]$; IR (KBr): $3319(\mathrm{NH}), 1594(\mathrm{C}=\mathrm{O}) \mathrm{cm}^{-1} ;{ }^{1} \mathrm{H}$ NMR $\left(300 \mathrm{MHz}, \mathrm{DMSO}-d_{6}\right): \delta 6.97(\mathrm{t}, J=7.3 \mathrm{~Hz}, 1 \mathrm{H}, \mathrm{Ar}), 7.20-7.35(\mathrm{~m}, 3 \mathrm{H}, \mathrm{Ar}), 7.40-7.52(\mathrm{~m}$, $3 \mathrm{H}, \mathrm{Ar}), 7.86(\mathrm{~d}, J=2.3 \mathrm{~Hz}, 1 \mathrm{H}, \mathrm{Ar}), 8.77\left(\mathrm{~s}, 1 \mathrm{H}, \mathrm{NH}\right.$, exch. $\left.\mathrm{D}_{2} \mathrm{O}\right), 8.96(\mathrm{~s}, 1 \mathrm{H}, \mathrm{NH}$, exch. $\left.\mathrm{D}_{2} \mathrm{O}\right) ;{ }^{13} \mathrm{C}$ NMR (125 MHz, DMSO- $\left.d_{6}\right): \delta 118.8$ (1C), 118.9 (2C), 119.7 (1C), 122.7 (1C), 123.5 (1C), $129.2(2 \mathrm{C}), 131.0$ (1C), 131.5 (1C), 139.7 (1C), 140.4 (1C), 152.7 (1C). Anal. calcd for $\mathrm{C}_{13} \mathrm{H}_{10} \mathrm{~N}_{2} \mathrm{OCl}_{2} \cdot 0.25 \mathrm{H}_{2} \mathrm{O}$ (285.43)\%:C 54.66; H 3.71; N 9.81. Found\%: C 54.99; H 3.55; N 9.86 .

1-(4-Chlorophenyl)-3-phenylurea (1cf, MCC) [40]. Prepared from 4-CA and phenyl isocyanate. Yield: $90 \%$ (white crystals): $\mathrm{mp} 228-229^{\circ} \mathrm{C}\left(\right.$ acetone $\left./ \mathrm{CHCl}_{3}\right) ; \mathrm{LC} / \mathrm{MS}(70 \mathrm{eV})$ $m / z(\%): 269\left[\mathrm{M}^{+},+23\right]$; IR (KBr): $3302(\mathrm{NH}), 1636(\mathrm{C}=\mathrm{O}) \mathrm{cm}^{-1} ;{ }^{1} \mathrm{H}$ NMR $(300 \mathrm{MHz}$, DMSO- $\left.d_{6}\right): \delta 6.95(\mathrm{t}, J=7.3 \mathrm{~Hz}, 1 \mathrm{H}, \mathrm{Ar}), 7.23-7.32(\mathrm{~m}, 4 \mathrm{H}, \mathrm{Ar}), 7.41-7.49(\mathrm{~m}, 4 \mathrm{H}, \mathrm{Ar}), 8.67(\mathrm{~s}$, 
$1 \mathrm{H}, \mathrm{NH}$, exch. $\left.\mathrm{D}_{2} \mathrm{O}\right), 8.86\left(\mathrm{~s}, 1 \mathrm{H}, \mathrm{NH}\right.$, exch. $\left.\mathrm{D}_{2} \mathrm{O}\right) ;{ }^{13} \mathrm{C}$ NMR (125 MHz, DMSO- $\left.d_{6}\right): \delta 118.8$ (2C), 120.2 (2C), 122.4 (1C), 125.8 (2C), 129.0 (1C), 129.2 (2C), 139.2 (1C), 139.9 (1C), 152.9 (1C). Anal. calcd for $\mathrm{C}_{13} \mathrm{H}_{11} \mathrm{~N}_{2} \mathrm{OCl}(246.58) \%: \mathrm{C} 63.29 ; \mathrm{H} 4.49 ; \mathrm{N} 11.36$. Found\%: $\mathrm{C}$ 63.67; H 4.50; N 11.40 .

1-(4-Chlorophenyl)-3-(4-chlorophenyl)urea (1cc, DCC) [41]. Prepared from 4-CA and 4-chlorophenyl isocyanate. Yield: $86 \%$ (pinkish solid): $\mathrm{mp} 223-224{ }^{\circ} \mathrm{C} ; \mathrm{LC} / \mathrm{MS}(70 \mathrm{eV}) \mathrm{m} / \mathrm{z}$ (\%): $281\left[\mathrm{M}^{+}\right]$; IR ( $\left.\mathrm{KBr}\right): 3296(\mathrm{NH}), 1651(\mathrm{C}=\mathrm{O}) \mathrm{cm}^{-1} ;{ }^{1} \mathrm{H}$ NMR spectrum was in agreement with the literature [42]. Anal. calcd for $\mathrm{C}_{13} \mathrm{H}_{10} \mathrm{ClN}_{2} \mathrm{O} \cdot 0.25 \mathrm{H}_{2} \mathrm{O}(285.64) \%$ : $\mathrm{C} 54.66 ; \mathrm{H} 3.71$; N 9.81. Found\%: C 54.85; H 3.51; N 9.80.

1-(3,4-Dichlorophenyl)-3-(2-methylphenyl)urea (1eg). Prepared from 3,4-dichloroaniline and o-tolyl isocyanate. Yield: $81 \%$ (white needle-shaped fine crystals): $\mathrm{mp} 217-218{ }^{\circ} \mathrm{C}$ (acetone $/ \mathrm{CHCl}_{3} / \mathrm{MeOH}$ ); LC/MS (70 eV) $\mathrm{m} / z$ (\%): 295 [M $\mathrm{M}^{+}$; IR (KBr): $3294(\mathrm{NH}), 1643$ $(\mathrm{C}=\mathrm{O}) \mathrm{cm}^{-1} ;{ }^{1} \mathrm{H}$ NMR $\left(500 \mathrm{MHz}, \mathrm{DMSO}-d_{6}\right): \delta 3.32\left(\mathrm{~s}, 3 \mathrm{H}, \mathrm{CH}_{3}\right), 6.96(\mathrm{t}, J=7.8 \mathrm{~Hz}, 1 \mathrm{H}, \mathrm{Ar})$, 7.12-7.17 (m, 2H, Ar), 7.27-7.29 (m, 1H, Ar), $7.49(\mathrm{~d}, J=8.8 \mathrm{~Hz}, 1 \mathrm{H}, \mathrm{Ar}), 7,74(\mathrm{~d}, J=7.8 \mathrm{~Hz}$, $1 \mathrm{H}, \mathrm{Ar}), 7.89(\mathrm{~d}, J=2.4 \mathrm{~Hz}, 1 \mathrm{H}, \mathrm{Ar}), 8.00\left(\mathrm{~s}, 1 \mathrm{H}, \mathrm{NH}\right.$ exch. $\left.\mathrm{D}_{2} \mathrm{O}\right), 9.27\left(\mathrm{~s}, 1 \mathrm{H}, \mathrm{NH}\right.$ exch. $\left.\mathrm{D}_{2} \mathrm{O}\right)$; ${ }^{13} \mathrm{C}$ NMR $\left(125 \mathrm{MHz}\right.$, DMSO- $\left.d_{6}\right): \delta 18.2(2 \mathrm{C}), 118.6(1 \mathrm{C}), 119.5$ (1C), $122.1(1 \mathrm{C}), 123.4(1 \mathrm{C})$, 126.6 (1C), 128.7 (1C), 130.7 (1C), 131.0 (1C), 131.5 (1C), 137.3 (1C), 140.5 (1C), 152.9 (1C). Anal. calcd for $\mathrm{C}_{14} \mathrm{H}_{12} \mathrm{Cl}_{2} \mathrm{~N}_{2} \mathrm{O}$ (295.14)\%: C 56.97; $\mathrm{H}$ 4.10; N 9.49. Found\%: C 57.03; $\mathrm{H}$ 4.19; N 9.47.

1-(3,4-Difluorophenyl)-3-(2-methylphenyl)urea (1gj). Prepared from 3,4-difluoroaniline and $o$-tolyl isocyanate. Yield: $79 \%$ (off-white crystals): $\mathrm{mp} 210-211{ }^{\circ} \mathrm{C}$ (acetone $/ \mathrm{CHCl}_{3}$ );

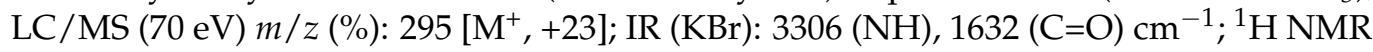
$\left(300 \mathrm{MHz}, \mathrm{DMSO}-d_{6}\right): \delta 2.21\left(\mathrm{~s}, 3 \mathrm{H}, \mathrm{CH}_{3}\right), 6.95(\mathrm{t}, J=7.3 \mathrm{~Hz}, 1 \mathrm{H}, \mathrm{Ar}), 7.05-7.17(\mathrm{~m}, 3 \mathrm{H}, \mathrm{Ar})$, 7.27-7.37 (m, 1H, Ar), 7.64-7.71 (m, 1H, Ar), $7.76(\mathrm{~d}, J=7.6 \mathrm{~Hz}, 1 \mathrm{H}, \mathrm{Ar}), 7.96(\mathrm{~s}, 1 \mathrm{H}, \mathrm{NH}$ exch. $\left.\mathrm{D}_{2} \mathrm{O}\right), 9.19\left(\mathrm{~s}, 1 \mathrm{H}, \mathrm{NH}\right.$ exch. $\left.\mathrm{D}_{2} \mathrm{O}\right) ;{ }^{13} \mathrm{C}$ NMR $\left(125 \mathrm{MHz}, \mathrm{DMSO}-d_{6}\right): \delta 18.3(1 \mathrm{C}), 107.4$ (1C), 114.5 (1C), 117.8 (1C), 121.9 (1C), 126.6 (1C), 123.5 (1C), 128.5 (1C), 130.7 (1C), 137.4 (1C), 137.5 (1C), $145.8(1 \mathrm{C}), 148.6(1 \mathrm{C}), 153.0(1 \mathrm{C})$. Anal. calcd for $\mathrm{C}_{14} \mathrm{H}_{12} \mathrm{~N}_{2} \mathrm{OF}_{2} \cdot 0.20 \mathrm{H}_{2} \mathrm{O}$ (265.95)\%: C 63.25; H 4.70; N 10.57. Found\%: C 63.02; H 4.62; N 10.41.

1-(3-Chloro-4-fluorophenyl)-3-(2-methylphenyl)urea (1gk). Prepared from 3-chloro-4fluoroaniline and $o$-tolyl isocyanate. Yield: $92 \%$ (off-white crystals): $\mathrm{mp} 210-211{ }^{\circ} \mathrm{C}$ (acetone $\left./ \mathrm{CHCl}_{3}\right)$; LC/MS (70 eV) $\mathrm{m} / z(\%)$ : $301\left[\mathrm{M}^{+},+23\right]$; IR (KBr): $3323(\mathrm{NH}), 1642(\mathrm{C}=\mathrm{O})$ $\mathrm{cm}^{-1}$; ${ }^{1} \mathrm{H}$ NMR $\left(300 \mathrm{MHz}, \mathrm{DMSO}-d_{6}\right): \delta 3.33\left(\mathrm{~s}, 3 \mathrm{H}, \mathrm{CH}_{3}\right), 6.95(\mathrm{t}, J=7.3 \mathrm{~Hz}, 1 \mathrm{H}, \mathrm{Ar})$, 7.10-7.20 (m, 2H, Ar), 7.22-7.34 (m, 2H, Ar), $7.75(\mathrm{~d}, J=8.3 \mathrm{~Hz}, 1 \mathrm{H}, \mathrm{Ar}), 7.82(\mathrm{dd}, J=6.4$, $2.3 \mathrm{~Hz}, 1 \mathrm{H}, \mathrm{Ar}), 7.96$ (s, exch. $\left.\mathrm{D}_{2} \mathrm{O}, 1 \mathrm{H}, \mathrm{NH}\right), 9.17$ (s, exch. $\left.\mathrm{D}_{2} \mathrm{O}, 1 \mathrm{H}\right) ;{ }^{13} \mathrm{C}$ NMR $(125 \mathrm{MHz}$, DMSO- $\left.d_{6}\right): \delta 16.9(1 \mathrm{C}), 113.6(1 \mathrm{C}), 114.6(1 \mathrm{C}), 120.9(1 \mathrm{C}), 123.6$ (1C), $124.2(1 \mathrm{C}), 125.9(1 \mathrm{C})$, 129.3 (1C), 131.7 (1C), 131.9 (1C), 134.2 (1C), 135.2 (1C), 152.9 (1C), 154.8 (1C). Anal. calcd for $\mathrm{C}_{14} \mathrm{H}_{12} \mathrm{~N}_{2} \mathrm{OFCl} \cdot 0.25 \mathrm{H}_{2} \mathrm{O}$ (282.56)\%: C 59.37; $\mathrm{H}$ 4.45; $\mathrm{N}$ 9.89. Found\%: C 59.62; $\mathrm{H}$ 4.36; N 9.84 .

1-(2-Metylphenyl)-3-(2-fluorophenyl)urea (1gl). Prepared from 2-fluoroaniline and otolyl isocyanate. Yield: quantitative yield (off-grey crystals); mp $224-225{ }^{\circ} \mathrm{C}$ (acetone);

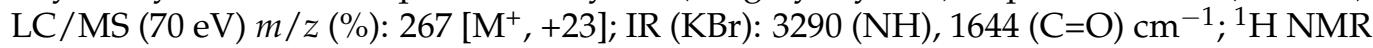
(300 MHz, DMSO- $\left.d_{6}\right): \delta 2.24\left(\mathrm{~s}, 3 \mathrm{H}, \mathrm{CH}_{3}\right), 6.91-7.09(\mathrm{~m}, 2 \mathrm{H}, \mathrm{Ar}), 7.11-7.25(\mathrm{~m}, 4 \mathrm{H}, \mathrm{Ar}), 7.84$ $(\mathrm{d}, J=7.6 \mathrm{~Hz}, 1 \mathrm{H}, \mathrm{Ar}), 8.18(\mathrm{dt}, J=8.6,1.5 \mathrm{~Hz}, 1 \mathrm{H}, \mathrm{Ar}), 8.34\left(\mathrm{~s}, 1 \mathrm{H}, \mathrm{NH}\right.$ exch. $\left.\mathrm{D}_{2} \mathrm{O}\right), 8.96(\mathrm{br}$ s, $1 \mathrm{H}, \mathrm{NH}$ exch. $\left.\mathrm{D}_{2} \mathrm{O}\right) ;{ }^{13} \mathrm{C}$ NMR $(125 \mathrm{MHz}, \mathrm{DMSO}-d 6): \delta 18.4(1 \mathrm{C}), 115.3(\mathrm{~d}, \mathrm{JCF}=18.9 \mathrm{~Hz}$, 1C), $120.9(1 \mathrm{C}), 121.6(1 \mathrm{C}), 122.6(\mathrm{~d}, \mathrm{JCF}=7.5 \mathrm{~Hz}, 1 \mathrm{C}), 123.3(1 \mathrm{C}), 124.9(1 \mathrm{C}), 126.6(1 \mathrm{C}), 128.0$ (1C), $128.2(1 \mathrm{C}), 130.7(1 \mathrm{C}), 137.6(1 \mathrm{C}), 152.3(\mathrm{~d}, \mathrm{JCF}=241.3 \mathrm{~Hz}, 1 \mathrm{C}), 152.9(1 \mathrm{C})$. Anal. calcd for $\mathrm{C}_{14} \mathrm{H}_{12} \mathrm{~N}_{2} \mathrm{OF}(244.26) \%$ : C 68.84; $\mathrm{H}$ 5.36; N 11.47. Found\%: C 68.74; H 5.34; N 11.37.

1-(3,4-Difluorophenyl)-3-phenylurea (1fj). Prepared from 3,4-difluoroaniline and phenyl isocyanate. Yield: $58 \%$ (greyish crystals): $\mathrm{mp} 213-214{ }^{\circ} \mathrm{C}$ (acetone); LC/MS (70 eV) $\mathrm{m} / \mathrm{z}$ (\%): $271\left[\mathrm{M}^{+},+23\right]$; IR (KBr): $3298(\mathrm{NH}), 1630(\mathrm{C}=\mathrm{O}) \mathrm{cm}^{-1} ;{ }^{1} \mathrm{H}$ NMR (500 MHz, DMSO- $\left.d_{6}\right)$ : $\delta 6.87(\mathrm{t}, J=7.6 \mathrm{~Hz}, 1 \mathrm{H}, \mathrm{Ar}), 7.05-7.15(\mathrm{~m}, 1 \mathrm{H}, \mathrm{Ar}), 7.24-7.32(\mathrm{~m}, 3 \mathrm{H}, \mathrm{Ar}), 7.42(\mathrm{~d}, J=7.3 \mathrm{~Hz}$, $2 \mathrm{H}, \mathrm{Ar}), 7.60-7.70(\mathrm{~m}, 1 \mathrm{H}, \mathrm{Ar}), 8.70$ (s, exch. $\left.\mathrm{D}_{2} \mathrm{O}, 1 \mathrm{H}, \mathrm{NH}\right), 8.85$ (s, $1 \mathrm{H}$, exch. $\left.\mathrm{D}_{2} \mathrm{O}, \mathrm{NH}\right) ;{ }^{13} \mathrm{C}$ NMR $\left(125 \mathrm{MHz}, \mathrm{DMSO}-d_{6}\right): \delta 107.6(\mathrm{~d}, J \mathrm{CF}=21.9 \mathrm{~Hz}, 1 \mathrm{C}), 114.8(\mathrm{dd}, J \mathrm{CF}=5.6,3.7 \mathrm{~Hz}, 1 \mathrm{C})$, $117.8(\mathrm{~d}, J \mathrm{CF}=1.7 \mathrm{~Hz}, 1 \mathrm{C}), 118.9(1 \mathrm{C}), 122.5(1 \mathrm{C}), 129.2(1 \mathrm{C}), 137.3(\mathrm{dd}, J \mathrm{CF}=9.5,2.8 \mathrm{~Hz}$, 
1C), $139.8(1 \mathrm{C}), 143.8(\mathrm{~d}, J \mathrm{CF}=12.4 \mathrm{~Hz}, 1 \mathrm{C}), 145.8(\mathrm{~d}, \mathrm{JCF}=12.4 \mathrm{~Hz}, 1 \mathrm{C}), 148.5(\mathrm{~d}, \mathrm{JCF}=13.3$ $\mathrm{Hz}, 1 \mathrm{C}), 150.5(\mathrm{~d}, \mathrm{JCF}=13.4 \mathrm{~Hz}, 1 \mathrm{C}), 152.9(1 \mathrm{C})$. Anal. calcd for $\mathrm{C}_{13} \mathrm{H}_{10} \mathrm{~N}_{2} \mathrm{OF}_{2}(248.23) \%$ : C 62.90; H 4.06; N 11.29. Found\%: C 62.61; H 4.08; N 11.19.

1-(3,4-Dichlorophenyl)-3-p-tolylurea (1ae) [43]. Prepared from $p$-toluidine and 3,4dichlorophenyl isocyanate. Yield: $21 \%$ (white fine spicules crystals): $\mathrm{mp} 225-226{ }^{\circ} \mathrm{C}$ (acetone $\left./ \mathrm{CHCl}_{3}\right)$; LC/MS (70 eV) m/z (\%): 317 [M+, +23]; IR (KBr): $3289(\mathrm{NH}), 1633(\mathrm{C}=\mathrm{O})$ $\mathrm{cm}^{-1} ;{ }^{1} \mathrm{H}$ NMR $\left(500 \mathrm{MHz}, \mathrm{DMSO}-d_{6}\right): \delta 2.06\left(\mathrm{~s}, 3 \mathrm{H}, \mathrm{CH}_{3}\right), 7.07(\mathrm{~d}, J=8.3 \mathrm{~Hz}, 2 \mathrm{H}, \mathrm{Ar})$, 7.28-7.32 (m, 3H, Ar), 7.48 (d, J = 8.8 Hz, 1H, Ar), 7.86 (d, J = 2.4 Hz, 1H, Ar), 8.65 (s, exch. $\left.\mathrm{D}_{2} \mathrm{O}, 1 \mathrm{H}, \mathrm{NH}\right), 8.91$ (s, exch. $\left.\mathrm{D}_{2} \mathrm{O}, 1 \mathrm{H}, \mathrm{NH}\right) ;{ }^{13} \mathrm{C}$ NMR (125 MHz, DMSO-d6): $\delta 20.8$ (1C), 118.7 (1C), 119.1 (1C), 119.6 (1C), 123.4 (2C), 129.6 (2C), 130.9 (1C), 131.5 (1C), 131.6 (1C), 137.1 (1C), 140.5 (1C), $152.8(1 \mathrm{C})$. Anal. calcd for $\mathrm{C}_{14} \mathrm{H}_{12} \mathrm{C}_{12} \mathrm{~N}_{2} \mathrm{O}(294.16) \%$ : C 56.97; $\mathrm{H} 4.10$; N 9.49. Found\%: C 56.56; H 4.09; N 9.36.

1-(3,4-Dichlorophenyl)-3-(4-methoxyl)phenylurea (1de) [44]. Prepared from 3,4-dichloroaniline and $p$-methoxylphenyl isocyanate. Yield: $97 \%$ (greyish crystals): mp $234-235{ }^{\circ} \mathrm{C}$ (acetone); LC/MS (70 eV) m/z (\%): 333 [M $\left.{ }^{+},+23\right]$; IR (KBr): $3263(\mathrm{NH}), 1644(\mathrm{C}=\mathrm{O}) \mathrm{cm}^{-1}$; ${ }^{1} \mathrm{H}$ NMR $\left(300 \mathrm{MHz}, \mathrm{DMSO}-d_{6}\right): \delta 3.69\left(\mathrm{~s}, 3 \mathrm{H}, \mathrm{CH}_{3}\right), 6.82-6.88(\mathrm{~m}, 2 \mathrm{H}, \mathrm{Ar}), 7.27-7.36$ (m, 3H, Ar), 7.47 $(\mathrm{d}, J=8.8 \mathrm{~Hz}, 1 \mathrm{H}, \mathrm{Ar}), 7.84(\mathrm{~d}, J=2.3 \mathrm{~Hz}, 1 \mathrm{H}, \mathrm{Ar}), 8.58(\mathrm{~s}, 1 \mathrm{H}, \mathrm{NH}), 8.89(\mathrm{~s}, 1 \mathrm{H}, \mathrm{NH}) ;{ }^{13} \mathrm{C}$ NMR (125 MHz, DMSO-d $_{6}$ : $\delta 55.6$ (1C), $114.4(2 \mathrm{C}), 119.6$ (1C), $120.4(1 \mathrm{C}), 120.9$ (2C), 123.4 (1C), 130.9 (1C), 131.5 (2C), 132.4 (1C), 140.4 (1C), 152.8 (1C), 155.2 (1C). Anal. calcd for $\mathrm{C}_{14} \mathrm{H}_{12} \mathrm{~N}_{2} \mathrm{O}_{2} \mathrm{Cl}_{2}$ (311.16)\%: C 54.04; H 3.89; N 9.00. Found\%: C 54.17; H 3.95; N 8.94.

\subsection{Antibacterial In Vitro Evaluation}

MICs $(\mu \mathrm{g} / \mathrm{mL})$ were determined by the broth microdilution method, using 96-well plates, according to CLSI [26]. Stock solutions of the investigated compounds were obtained by setting the concentration at the maximum possible value. Then, the stock solutions were diluted 1:10 with Mueller Hinton Broth (Oxoid, Italy). Afterwards, twofold serial dilutions in the suitable test medium were carried out to obtain a set of concentrations from 512 to $2 \mu \mathrm{g} / \mathrm{mL}$ in the wells. To obtain the stock solution, DMSO (100\%) was employed as diluent. The following bacteria strains, available as freeze-dried discs, belonging to the ATCC collection, were used: Gram-positive: S. aureus ATCC 29213, 6538, and 6538P, E. faecalis ATCC 29212; Gram-negative: K. pneuomoniae ATCC 13883, P. aeruginosa ATCC 27853. To conserve the purity of cultures and to enable their reproducibility, cryovials of all microbial strains in the medium were set up and stored at $-80{ }^{\circ} \mathrm{C}$. Pre-cultures of each bacterial strain were prepared in Mueller Hinton Broth (MHB) and incubated at $37^{\circ} \mathrm{C}$ for $3-5 \mathrm{~h}$. The turbidity of bacterial cell suspension was calibrated to $0.5 \mathrm{McFarland}$

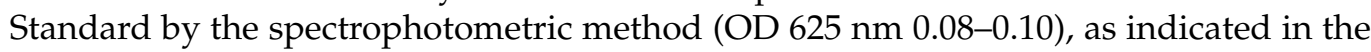
CLSI protocol M7-A9 and, further, the standardized suspension was diluted (1:100) with $\mathrm{MHB}$ to reach 1-2 $\times 10^{6} \mathrm{CFU} / \mathrm{mL}$. All wells were seeded with $100 \mu \mathrm{l}$ of the mentioned final inoculum, and some wells contained only inoculated broth as control growth. The plates were incubated at $37^{\circ} \mathrm{C}$ for $24 \mathrm{~h}$, and the MIC values were recorded as the lowest concentration of compounds at which there was no optically detectable microorganism growth and evaluated by comparing the growth in every well visually with that of the growth control well for bacteria. The MICs were determined by using the assay repeated twice in triplicate. Throughout the study, norfloxacin was used as reference antibiotic. Appropriate controls involved norfloxacin for bacteria as international reference standard. Additionally, DMSO alone (used as diluent of tested compounds) was tested for its activity against bacteria, and consequently, quantities of this solvent under $5 \%$ concentration were used during the execution of the experiments. Each species was tested at least three times in duplicate. The MBM assay for bacteria was based on the recommended procedures by CLSI [26].

\subsection{Cell Cultures}

Human MCF-7 and MDA-MB-231 breast cancer cells, HeLa and ISHIKAWA uterine carcinoma cells, A2058 melanoma epithelial cells, MCF-10A mammary epithelial cells and 
Hek-293 embryonic kidney epithelial cells were obtained from American Type Culture Collection (ATCC, Manassas, VA, USA). Estrogen receptor positive [(ER(+)] MCF-7 and triple negative MDA-MB-231 breast cancer cells were maintained in Dulbecco's modified Eagle's medium/nutrient mixture F-12 Ham (DMEM-F12) medium containing $2 \mathrm{mmol} / \mathrm{L}$ L-glutamine, $1 \mathrm{mg} / \mathrm{mL}$ penicillin-streptomycin and supplemented with $5 \%$ fetal bovine serum (FBS) [45]. HeLa epithelial cervix carcinoma cells, estrogen receptor negative [ER(-)], and Ishikawa endometrial adenocarcinoma cell, estrogen receptor positive [ER(+)], were kept in minimum essential medium (MEM), supplemented with $10 \%$ FBS, $100 \mathrm{U} / \mathrm{mL}$ penicillin/streptomycin and 1\% non-essential amino acid. A2058 highly metastatic melanoma epithelial cells were kept in DMEM low glucose $(1 \mathrm{~g} / \mathrm{L})$, supplemented with $20 \%$ FBS, $1 \%$ l-glutamine and $100 \mathrm{U} / \mathrm{mL}$ penicillin/streptomycin. MCF-10A mammary epithelial cells, were cultured in DMEM/F12 medium, supplemented with 5\% horse serum (HS), $100 \mathrm{U} / \mathrm{mL}$ penicillin/streptomycin, $0.5 \mathrm{mg} / \mathrm{mL}$ hydrocortisone, $20 \mathrm{ng} / \mathrm{mL}$ human epidermal growth factor (hEGF), $10 \mathrm{mg} / \mathrm{mL}$ insulin and $0.1 \mathrm{mg} / \mathrm{mL}$ cholera enterotoxin (Sigma-Aldrich, Milan, Italy). Hek-293 embryonic kidney epithelial cells were cultured in DMEM high glucose $(4.5 \mathrm{~g} / \mathrm{L})$, supplemented with $10 \% \mathrm{FBS}, 1 \%$ l-glutamine and $100 \mathrm{U} / \mathrm{mL}$ penicillin/streptomycin. Cells were conserved at $37^{\circ} \mathrm{C}$ in a humid atmosphere of $95 \%$ air and $5 \% \mathrm{CO}_{2}$, and screened for contamination at regular intervals $[46,47]$.

\subsection{Cell Viability}

Cell viability was determined using the 3-(4,5-dimethylthiazol-2-y1)-2,5-diphenyltetrazolium bromide (MTT, Sigma-Aldrich, Milan, Italy) assay [48,49]. Cells were seeded on 48-well plates and grown in complete medium. Before treatment, cells were starved in serum free medium for $24 \mathrm{~h}$ to allow cell cycle synchronization. Then, cells were treated in phenol-red-free medium supplemented with $1 \%$ of serum with increasing concentrations of each compound for $72 \mathrm{~h}$; then, fresh MTT, re-suspended in phosphate-buffer saline (PBS), was added to each well (final concentration $\left(0.5 \mathrm{mg} / \mathrm{mL}\right.$ ). After $2 \mathrm{~h}$ incubation at $37^{\circ} \mathrm{C}$, cells were lysed with DMSO, and then optical density was measured at $570 \mathrm{~nm}$ using a microplate reader. For each sample, the mean absorbance was expressed as a percentage over the control and plotted versus drug concentrations to determine the $\mathrm{IC}_{50}$ values (i.e., drug concentrations able to decrease cell viability by $50 \%$ with respect to control) for each cell line, using GraphPad Prism 8 software (GraphPad Inc., San Diego, CA, USA). Data are representative of three independent experiments; standard deviations (SD) are shown.

\section{Conclusions}

In the search for new compounds endowed with antibacterial activity but with more favorable toxicological properties than TCC, a series of diarylureas were synthesized. Structure-activity relationship studies showed that the 3,4-disubstitution pattern of TCC seems to be not essential for antibacterial activity. Indeed, the highest antimicrobial activity against $S$. aureus was found for compounds bearing a 2,6-xylyl moiety. Particularly, compounds $1 \mathrm{ab}$ and $\mathbf{1 b c}$ were the most interesting of the series showing the same activity as TCC against $S$. aureus and an even higher activity than TCC against $E$. faecalis. These compounds also displayed no cytotoxicity against MCF-10A and Hek-293 cell lines. Based on their simple preparation and interesting antibacterial activity profile, the newly prepared small molecules are promising candidates for antibacterial drug development to be introduced in personal care products in substitution of TCC.

Author Contributions: Writing-Original Draft Preparation, A.C.; Data Curation, D.I.; Validation, A.R.; Methodology, L.S., J.C. and F.L.; Writing—Review and Editing, C.F.; Supervision, M.S.S. All authors have read and agreed to the published version of the manuscript.

Funding: This study was supported by MIUR of Italy (ex 60\%).

Data Availability Statement: The data presented in this study are available in manuscript.

Conflicts of Interest: The authors declare no conflict of interest. 


\begin{abstract}
Abbreviations
4-CA:4-Chloroaniline; CEC, Contaminant of Emerging Concern; CLSI, Clinical and Laboratory Standards Institute; DCC, 4,4'-Dichlorocarbanilide; DMSO, Dimethyl sulfoxide; hEGF, Human epidermal growth factor; FBS, Fetal bovine serum; HS, Horse serum; IR, Infrared; LC-MS, Liquid chromatography-mass spectrometry; MCC, 1-(3-Chlorophenyl)-3-phenylurea; MICs, Minimum Inhibitory Concentrations; MEM, Minimum essential medium; MTT, 3-(4,5-Dimethylthiazol-2-y1)-2,5diphenyltetrazolium bromide; NCC, Carbanilide; NMR, Nuclear Magnetic Resonance; SD, Standard deviations; TCC, Triclocarban.
\end{abstract}

\title{
References
}

1. Halden, R.U.; Paull, D.H. Response to Comment On "Co-Occurrence of Triclocarban and Triclosan in U.S. Water Resources." . Environ. Sci. Technol. 2005, 39, 6335-6336. [CrossRef]

2. Halden, R.U.; Lindeman, A.E.; Aiello, A.E.; Andrews, D.; Arnold, W.A.; Fair, P.; Fuoco, R.E.; Geer, L.A.; Johnson, P.I.; Lohmann, R.; et al. The Florence Statement on Triclosan and Triclocarban. Environ. Health Perspect. 2017, 125, 064501. [CrossRef]

3. Halden, R.U. On the Need and Speed of Regulating Triclosan and Triclocarban in the United States. Environ. Sci. Technol. 2014, 48, 3603-3611. [CrossRef] [PubMed]

4. Catalano, A.; Iacopetta, D.; Sinicropi, M.S.; Franchini, C. Diarylureas as Antitumor Agents. Appl. Sci. 2021, 11, 374. [CrossRef]

5. Catalano, A.; Iacopetta, D.; Pellegrino, M.; Aquaro, S.; Franchini, C.; Sinicropi, M. Diarylureas: Repositioning from Antitumor to Antimicrobials or Multi-Target Agents against New Pandemics. Antibiotics 2021, 10, 92. [CrossRef]

6. Catalano, A. COVID-19: Could Irisin Become the Handyman Myokine of the 21st Century? Coronaviruses 2020, 1, 32-41. [CrossRef]

7. Rochester, J.R.; Bolden, A.L.; Pelch, K.E.; Kwiatkowski, C.F. Potential Developmental and Reproductive Impacts of Triclocarban: A Scoping Review. J. Toxicol. 2017, 2017, 1-15. [CrossRef] [PubMed]

8. Musee, N. Environmental risk assessment of triclosan and triclocarban from personal care products in South Africa. Environ. Pollut. 2018, 242, 827-838. [CrossRef] [PubMed]

9. Taweetanawanit, P.; Ratpukdi, T.; Siripattanakul-Ratpukdi, S. Performance and kinetics of triclocarban removal by entrapped Pseudomonas fluorescens strain MC46. Bioresour. Technol. 2019, 274, 113-119. [CrossRef]

10. Kor-Bicakci, G.; Abbott, T.; Ubay-Cokgor, E.; Eskicioglu, C. Occurrence and fate of antimicrobial triclocarban and its transformation products in municipal sludge during advanced anaerobic digestion using microwave pretreatment. Sci. Total. Environ. 2020, 705, 135862. [CrossRef]

11. Silveira, R.S.; Rocha, B.A.; Rodrigues, J.L.; Barbosa, F. Rapid, sensitive and simultaneous determination of 16 endocrine-disrupting chemicals (parabens, benzophenones, bisphenols, and triclocarban) in human urine based on microextraction by packed sorbent combined with liquid chromatography tandem mass spectrometry (MEPS-LC-MS/MS). Chemosphere 2020, 240, 124951. [CrossRef] [PubMed]

12. Gao, C.-J.; Kannan, K. Phthalates, bisphenols, parabens, and triclocarban in feminine hygiene products from the United States and their implications for human exposure. Environ. Int. 2020, 136, 105465. [CrossRef]

13. Liang, B.; Yun, H.; Kong, D.; Ding, Y.; Li, X.; Vangnai, A.S.; Wang, A. Bioaugmentation of triclocarban and its dechlorinated congeners contaminated soil with functional degraders and the bacterial community response. Environ. Res. 2020, 180, 108840. [CrossRef] [PubMed]

14. Yun, H.; Liang, B.; Kong, D.; Li, X.; Wang, A. Fate, risk and removal of triclocarban: A critical review. J. Hazard. Mater. 2020, 387, 121944. [CrossRef]

15. Food and Drug Administration. Safety and effectiveness of consumer antiseptics: topical antimicrobial drug products for over-the-counter human use. Final rule. Fed. Regist. 2016, 81, 61106-61130.

16. Brose, D.A.; Kumar, K.; Liao, A.; Hundal, L.S.; Tian, G.; Cox, A.; Zhang, H.; Podczerwinski, E.W. A reduction in triclosan and triclocarban in water resource recovery facilities' influent, effluent, and biosolids following the U.S. Food and Drug Administration's 2013 proposed rulemaking on antibacterial products. Water Environ. Res. 2019, 91, 715-721. [CrossRef] [PubMed]

17. Arifin, S.N.H.; Mohamed, R.; Al-Gheethi, A.; Lai, C.W.; Yashni, G. Heterogeneous photocatalysis of triclocarban and triclosan in greywater: a systematic and bibliometric review analysis. Int. J. Environ. Anal. Chem. 2021, 1-19. [CrossRef]

18. Pujol, E.; Blanco-Cabra, N.; Julián, E.; Leiva, R.; Torrents, E.; Vázquez, S. Pentafluorosulfanyl-containing Triclocarban Analogs with Potent Antimicrobial Activity. Molecules 2018, 23, 2853. [CrossRef]

19. Qiao, L.; Hao, S. Novel Trifluoromethylcoumarinyl Urea Derivatives: Synthesis, Characterization, Fluorescence, and Bioactivity. Molecules 2018, 23, 600. [CrossRef]

20. Lipinski, C.A.; Lombardo, F.; Dominy, B.W.; Feeney, P.J. Experimental and computational approaches to estimate solubili-ty and permeability in drug discovery and development settings. Adv. Drug Deliv. Rev. 1997, 23, 3-25. [CrossRef] 
21. Catalano, A.; Carocci, A.; Corbo, F.; Franchini, C.; Muraglia, M.; Scilimati, A.; De Bellis, M.; De Luca, A.; Camerino, D.C.; Sinicropi, M.S.; et al. Constrained analogues of tocainide as potent skeletal muscle sodium channel blockers towards the development of antimyotonic agents. Eur. J. Med. Chem. 2008, 43, 2535-2540. [CrossRef]

22. De Luca, A.; Talon, S.; De Bellis, M.; Desaphy, J.-F.; Franchini, C.; Lentini, G.; Catalano, A.; Corbo, F.; Tortorella, V.; ConteCamerino, D. Inhibition of skeletal muscle sodium currents by mexiletine analogues: specific hydrophobic interactions rather than lipophilia per se account for drug therapeutic profile. Naunyn-Schmiedeberg's Arch. Pharmacol. 2003, 367, 318-327. [CrossRef]

23. Bruno, C.; Carocci, A.; Catalano, A.; Cavalluzzi, M.M.; Corbo, F.; Franchini, C.; Lentini, G.; Tortorella, V. Facile, alternative route to Lubeluzole, its enantiomer, and the racemate. Chirality 2006, 18, 227-231. [CrossRef]

24. Raimondi, M.V.; Presentato, A.; Petri, G.L.; Buttacavoli, M.; Ribaudo, A.; De Caro, V.; Alduina, R.; Cancemi, P. New Synthetic Nitro-Pyrrolomycins as Promising Antibacterial and Anticancer Agents. Antibiotics 2020, 9, 292. [CrossRef]

25. Kaymakçioğlu, B.K.; Rollas, S.; Körceğez, E.; Aricioğlu, F.; Kaymakçığlu, B.K.; Arıcıoğlu, F. Synthesis and biological evaluation of new N-substituted-N'-(3,5-di/1,3,5-trimethylpyrazole-4-yl)thiourea/urea derivatives. Eur. J. Pharm. Sci. 2005, 26, 97-103. [CrossRef] [PubMed]

26. CLSI. Methods for Dilution Antimicrobial Susceptibility Tests for Bacteria that Grow Aerobically. Approved [document M7-A9], CLSI; Clinical and Laboratory Standards Institute: Wayne, PA, USA, 2012.

27. Pozzi, C.; Ferrari, S.; Cortesi, D.; Luciani, R.; Stroud, R.M.; Catalano, A.; Costi, M.P.; Mangani, S. The structure ofEnterococcus faecalisthymidylate synthase provides clues about folate bacterial metabolism. Acta Crystallogr. Sect. D Biol. Crystallogr. 2012, 68, 1232-1241. [CrossRef] [PubMed]

28. Catalano, A.; Luciani, R.; Carocci, A.; Cortesi, D.; Pozzi, C.; Borsari, C.; Ferrari, S.; Mangani, S. X-ray crystal structures of Enterococcus faecalis thymidylate synthase with folate binding site inhibitors. Eur. J. Med. Chem. 2016, 123, 649-664. [CrossRef]

29. Kao, P.H.N.; Kline, K.A. Dr. Jekyll and Mr. Hide: How Enterococcus faecalis subverts the host immune response to cause infection. J. Mol. Biol. 2019, 431, 2932-2945. [CrossRef] [PubMed]

30. Kim, S.; Rhee, M. Microbicidal effects of plain soap vs triclocarban-based antibacterial soap. J. Hosp. Infect. 2016, 94, 276-280. [CrossRef]

31. Zhu, T.-H.; Xu, X.-P.; Cao, J.-J.; Wei, T.-Q.; Wang, S.-Y.; Ji, S.-J. Cobalt(II)-Catalyzed Isocyanide Insertion Reaction with Amines under Ultrasonic Conditions: A Divergent Synthesis of Ureas, Thioureas and Azaheterocycles. Adv. Synth. Catal. 2014, 356, 509-518. [CrossRef]

32. Malviya, B.K.; Jaiswal, P.K.; Verma, V.P.; Badsara, S.S.; Sharma, S. Electrochemical Synthesis of Carbodiimides via Metal/OxidantFree Oxidative Cross-Coupling of Amines and Isocyanides. Org. Lett. 2020, 22, 2323-2327. [CrossRef]

33. Wang, L.; Wang, H.; Wang, Y.; Shen, M.; Li, S. Photocatalyzed synthesis of unsymmetrical ureas via the oxidative decarboxylation of oxamic acids with PANI-g-C3N4-TiO2 composite under visible light. Tetrahedron Lett. 2020, 61, 151962. [CrossRef]

34. Kumar, A.; Kumar, N.; Sharma, R.; Bhargava, G.; Mahajan, D. Direct Conversion of Carboxylic Acids to Various NitrogenContaining Compounds in the One-Pot Exploiting Curtius Rearrangement. J. Org. Chem. 2019, 84, 11323-11334. [CrossRef]

35. Mozaffari, M.; Nowrouzi, N. Palladium-Catalyzed Synthesis of Symmetrical and Unsymmetrical Ureas Using Chromium Hexacarbonyl as a Convenient and Safe Alternative Carbonyl Source. Eur. J. Org. Chem. 2019, 46, 7541-7544. [CrossRef]

36. Kadam, S.A.; Haav, K.; Toom, L.; Haljasorg, T.; Leito, I. NMR Method for Simultaneous Host-Guest Binding Constant Measurement. J. Org. Chem. 2014, 79, 2501-2513. [CrossRef]

37. Singh, A.S.; Agrahari, A.K.; Singh, S.K.; Yadav, M.S.; Tiwari, V.K. An Improved Synthesis of Urea Derivatives from NAcylbenzotriazole via Curtius Rearrangement. Synthesis 2019, 51, 3443-3450. [CrossRef]

38. Inaloo, I.D.; Majnooni, S. A Fe $\mathrm{O}_{4} @ \mathrm{SiO}_{2}$ /Schiff Base/Pd Complex as an Efficient Heterogeneous and Recyclable Nanocatalyst for One-Pot Domino Synthesis of Carbamates and Unsymmetrical Ureas. Eur. J. Org. Chem. 2019, 2019, 6359-6368. [CrossRef]

39. Khan, K.M.; Saeed, S.; Ali, M.; Gohar, M.; Zahid, J.; Khan, A.; Perveen, S.; Choudhary, M.I. Unsymmetrically disubstituted urea derivatives: A potent class of antiglycating agents. Bioorganic Med. Chem. 2009, 17, 2447-2451. [CrossRef]

40. Bao, J.; Kuik, D.; Tranmer, G.K. An efficient one-pot synthesis of N, N'-disubstituted phenylureas and N-aryl carbamates using hydroxylamine-O-sulfonic acid. Tetrahedron 2018, 74, 5546-5553. [CrossRef]

41. Chen, B.; Peng, J.-B.; Ying, J.; Qi, X.; Wu, X.-F. A Palladium-Catalyzed Domino Procedure for the Synthesis of Unsymmetrical Ureas. Adv. Synth. Catal. 2018, 360, 2820-2824. [CrossRef]

42. Azarifar, D.; Golbaghi, M. Selective and facile oxidative desulfurization of thioureas and thiobarbituric acids with singlet molecular oxygen generated from trans-3,5-dihydroperoxy-3,5-dimethyl-1,2-dioxolane. J. Sulfur Chem. 2015, 37, 1-13. [CrossRef]

43. Zhang, Y.; Anderson, M.; Weisman, J.L.; Lu, M.; Choy, C.J.; Boyd, V.A.; Price, J.; Sigal, M.; Clark, J.; Connelly, M.; et al. Evaluation of Diarylureas for Activity Against Plasmodium falciparum. ACS Med. Chem. Lett. 2010, 1, 460-465. [CrossRef]

44. Yao, H.; Liu, F.; Chen, J.; Li, Y.; Cui, J.; Qiao, C. Antischistosomal activity of N,N'-arylurea analogs against Schistosoma japonicum. Bioorganic Med. Chem. Lett. 2016, 26, 1386-1390. [CrossRef]

45. Sirignano, E.; Saturnino, C.; Botta, A.; Sinicropi, M.S.; Caruso, A.; Pisano, A.; Lappano, R.; Maggiolini, M.; Longo, P. Synthesis, characterization and cytotoxic activity on breast cancer cells of new half-titanocene derivatives. Bioorganic Med. Chem. Lett. 2013, 23, 3458-3462. [CrossRef]

46. Iacopetta, D.; Grande, F.; Caruso, A.; Mordocco, R.A.; Plutino, M.R.; Scrivano, L.; Ceramella, J.; Muià, N.; Saturnino, C.; Puoci, F.; et al. New insights for the use of quercetin analogs in cancer treatment. Future Med. Chem. 2017, 9, 2011-2028. [CrossRef] 
47. Iacopetta, D.; Carocci, A.; Sinicropi, M.S.; Catalano, A.; Lentini, G.; Ceramella, J.; Curcio, R.; Caroleo, M.C. Old Drug Scaffold, New Activity: Thalidomide-Correlated Compounds Exert Different Effects on Breast Cancer Cell Growth and Progression. ChemMedChem 2017, 12, 381-389. [CrossRef]

48. Ceramella, J.; Caruso, A.; Occhiuzzi, M.A.; Iacopetta, D.; Barbarossa, A.; Rizzuti, B.; Dallemagne, P.; Rault, S.; El-Kashef, H.; Saturnino, C.; et al. Benzothienoquinazolinones as new multi-target scaffolds: Dual inhibition of human Topoisomerase I and tubulin polymerization. Eur. J. Med. Chem. 2019, 181, 111583. [CrossRef]

49. Tundis, R.; Iacopetta, D.; Sinicropi, M.; Bonesi, M.; Leporini, M.; Passalacqua, N.; Ceramella, J.; Menichini, F.; Loizzo, M. Assessment of antioxidant, antitumor and pro-apoptotic effects of Salvia fruticosa Mill. subsp. thomasii (Lacaita) Brullo, Guglielmo, Pavone \& Terrasi (Lamiaceae). Food Chem. Toxicol. 2017, 106, 155-164. [CrossRef] 